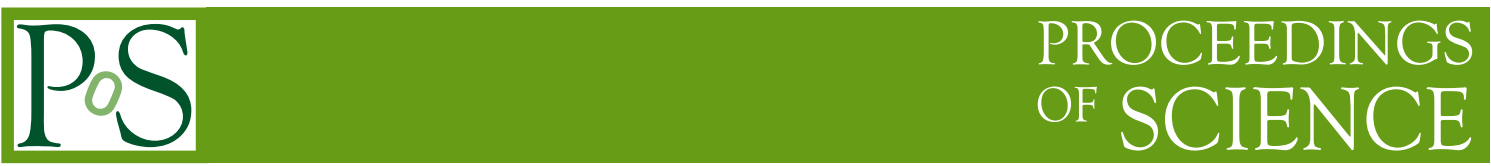

\title{
The ALICE Inner Tracking System Upgrade
}

\author{
Sabyasachi Siddhanta*† \\ Department of Physics, University of Cagliari and INFN Cagliari, Cagliari, Italy \\ E-mail: sabyasachi.siddhantalcern.ch
}

The long term plan of ALICE (A Large Ion Collider Experiment) at the CERN Large Hadron Collider (LHC) is a detailed investigation and characterisation of the Quark-Gluon Plasma (QGP). ALICE has devised a comprehensive upgrade strategy to enhance its physics capabilities and to exploit the LHC running conditions after the second long shutdown of the LHC scheduled in 2019-20. The upgraded ALICE will focus on high precision measurements of rare probes over a wide range of momenta, which will significantly improve the performance with respect to the present experimental set up. The upgrade strategy is based on the fact that after LS2 LHC will progressively increase its luminosity with $\mathrm{Pb}$ beams eventually reaching an interaction rate of about $50 \mathrm{kHz}$. To exploit the new LHC capabilities, several existing detectors will undergo a substantial upgrade and new detectors will be added. Within this upgrade strategy, the Inner Tracking System (ITS) upgrade forms an important cornerstone, providing precise measurements for heavy-flavour interactions with the QGP medium and for the production of thermal photons and low-mass dileptons. The present ITS will be replaced by a new seven-layer ITS consisting of Monolithic Active Pixel Sensors (MAPS) with highly improved tracking and vertexing abilities. In this contribution, an overview of the ITS upgrade, the technical developments for the detector elements and some performance studies are presented.

7th International Conference on Physics and Astrophysics of Quark Gluon Plasma

1-5 February, 2015

Kolkata, India

${ }^{*}$ Speaker.

${ }^{\dagger}$ on behalf of the ALICE collaboration. 


\section{Introduction}

ALICE (A Large Ion Collider Experiment) at the CERN LHC studies the physics of strongly interacting matter at unprecedented energy densities using heavy ion collisions [1]. Its long term goal is a detailed study of the Quark-Gluon Plasma (QGP), utilizing the scientific potential of the LHC with $\mathrm{Pb}$ ions after the second long shutdown (LS2, 2019-20). ALICE has devised an upgrade strategy $[2,3]$ to enhance its capabilities after LS2. The aim is to read-out all $\mathrm{Pb}-\mathrm{Pb}$ interactions $(50$ $\mathrm{kHz}$ ) and accumulate more than $10 \mathrm{nb}^{-1}$ of $\mathrm{Pb}-\mathrm{Pb}$ collisions following LS2, gaining a factor of 100 in statistics for minimum bias trigger over the current programme up to LS2. In this strategy, the upgrade of the Inner Tracking System (ITS) forms an important part which will provide improved vertexing and tracking capabilities in the central rapidity region for new measurements with focus on the production of heavy flavour, low-mass dileptons and thermal photons.

\section{Design motivation and specifications for the Inner Tracking System upgrade}

The upgraded ITS should provide significant performance improvement, mainly in terms of: the impact parameter resolution (by at least a factor of 3 below $p_{\mathrm{T}}=1 \mathrm{GeV} / c$ with respect to the present ITS), standalone tracking capability (particularly at $p_{\mathrm{T}}<1 \mathrm{GeV} / c$ ) and readout capabilities (to readout $50 \mathrm{kHz} \mathrm{Pb}-\mathrm{Pb}$ interactions). The present ITS, consisting of six layers of silicon detectors (two layers each of Silicon Pixel Detectors, Silicon Drift Detectors and Silicon Strip Detectors), will be replaced by a new ITS with seven layers of silicon pixel detectors [4]. The three innermost layers and the four outermost layers constitute the Inner Barrel (IB) and the Outer Barrel (OB) respectively, as shown in the left panel of Fig. 1. The position of the layers are optimized to improve the tracking efficiency, $p_{\mathrm{T}}$ resolution and impact parameter resolution. The radius of the first layer is reduced from $39 \mathrm{~mm}$ in the present ITS to $22 \mathrm{~mm}$ along with a reduction of the beam pipe radius from the present $29 \mathrm{~mm}$ to $18.2 \mathrm{~mm}$ in the upgrade scenario. The upgraded ITS targets an effective material budget of $0.3 \%$ of the radiation length for the IB (compared to $1.14 \%$ for the first two layers in the current ITS) and $1.0 \%$ of the radiation length for the OB. The objective is to have a spatial resolution of $5 \mu \mathrm{m}$ in both $r-\phi$ and $z$ directions for all the layers.

\section{Pixel technology, prototype sensors and their performance}

Recent advancements in the field of Monolithic Active Pixel Sensors (MAPS) led to the selection of these devices for the ALICE ITS upgrade. The ULTIMATE chip used in the STAR PXL detector, which is an upgrade to the STAR detector at RHIC [5] is the first application of MAPS in a collider experiment. This chip didn't meet the ALICE ITS upgrade requirements, specially in terms of readout speed, radiation tolerance and power consumption limits. ALICE pursued a dedicated R \& D programme to develop a new pixel chip using the TowerJazz $0.18 \mu \mathrm{m}$ technology [6]. This technology provides important features like the availability of a deep p-well option which ushers the use of PMOS transistors on a p-type epitaxial layer without degrading the charge collection efficiency. Thus a full CMOS process can be used inside the pixel circuit allowing low-power in-pixel circuit designs. The right panel of Fig. 1 shows a schematic cross section of a pixel in this 

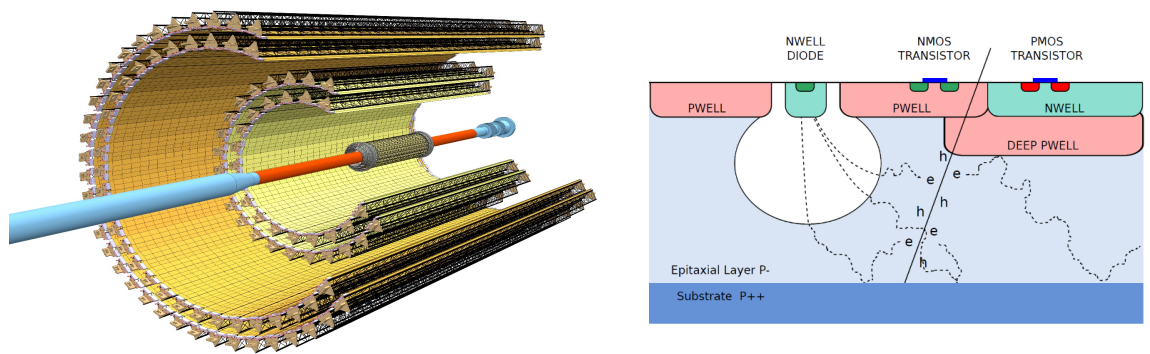

Figure 1: The left panel shows the layout of the upgraded ITS. The three innermost layers constitute the Inner Barrel and the four outermost layers constitute the Outer Barrel. The right panel shows the schematic cross section of a MAPS pixel in the TowerJazz $0.18 \mu \mathrm{m}$ technology with the deep p-well feature.

technology using a deep p-well. The technology also provides high resistivity $(>1 \mathrm{k} \Omega \mathrm{cm})$ epitaxial layers and the possibility to apply a reverse bias (up to $8 \mathrm{~V}$ ) on the substrate, which enhances the charge collection efficiency.

The requirements of the pixel chip for the upgrade [4] are mainly: low power $\left(<100 \mathrm{~mW} / \mathrm{cm}^{2}\right)$, low integration time $(<30 \mu \mathrm{s})$, high detection efficiency $(>99 \%)$, low fake hit rate $\left(<10^{-5}\right.$ per pixel and event) and sufficient radiation hardness (Total Ionisation Dose of $\sim 2700 \mathrm{krad}$ and a fluence of $\sim 1.7 \times 10^{13} 1 \mathrm{MeV} \mathrm{n}$ eq $/ \mathrm{cm}^{2}$ of Non Ionising Energy Loss). The pixel pitch should be in the order of $30 \mu \mathrm{m}$ and the silicon thickness about $50 \mu \mathrm{m}$.

ALICE has pursued two different design streams: ALPIDE [4, 7] and MISTRAL-O [4]. MISTRAL-O is derived from the STAR ULTIMATE chip and uses end-of-column discriminators. It uses a rolling shutter architecture with an integration time of $20 \mu \mathrm{s}$ and a power density of about $80 \mathrm{~mW} / \mathrm{cm}^{2}$. ALPIDE targets further reduction of the power density and it uses in-pixel discriminators. In ALPIDE, the pixel response is stored in local memories in presence of a global strobe signal and is fed into an encoder circuit that generates the address of a hit pixel [8]. This architecture has a power density of less than $35 \mathrm{~mW} / \mathrm{cm}^{2}$ for the inner layers and of about $17 \mathrm{~mW} / \mathrm{cm}^{2}$ for the outer layers and fast read-out time (event time resolution: $2 \mu \mathrm{s}$ ). ALPIDE serves as the baseline architecture for the upgrade. Several prototypes implementing different components of these architectures were validated. The Explorer series of (analogue -) prototypes of the ALPIDE family, for example, were fabricated to study the charge collection properties with different sensing node geometries using different starting materials.

The first full scale prototype of ALPIDE, pALPIDE-1, was fabricated in 2014. The chip size is $15 \mathrm{~mm}$ x $30 \mathrm{~mm}$ consisting of $512 \times 1024$ pixels, with a pixel-pitch of $28 \mu \mathrm{m}$. The chip is divided into four sectors consisting of different types of pixels, as indicated in the left panel of Fig. 2. They differ either in the reset mechanism of the pixels (reset using a PMOS transistor or a forward biased diode) or in the spacing between the collection diode and the surrounding p-well. Extensive tests with pALPIDE-1 were performed at different institutes and test beam facilites. A custom readout system (shown in the right panel of Fig. 2) was developed for testing pALPIDE-1. The readout board, based on an Altera Cyclone III FPGA, reads out the pALPIDE-1 (which is wire-bonded on a carrier board) and transfers the data to the user through a USB 3.0 interface.

Fig. 3 shows the excellent response of pALPIDE-1 (sector 2 with $-3 \mathrm{~V}$ reverse substrate bias) to $6 \mathrm{GeV} / c \pi^{-}$. The left panel shows the detection efficiency and fake hit rate of non-irradiated and 

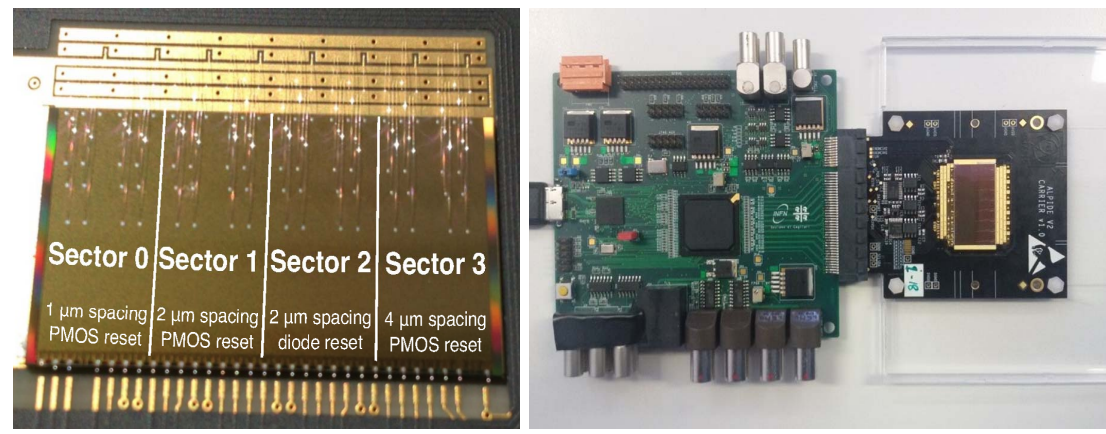

Figure 2: The left panel shows the pALPIDE-1 chip with the four sectors indicated. The right panel shows the custom readout system used for tests of the pALPIDE-1. The FPGA-based board reads out the pALPIDE1 which is wire-bonded on a carrier card.
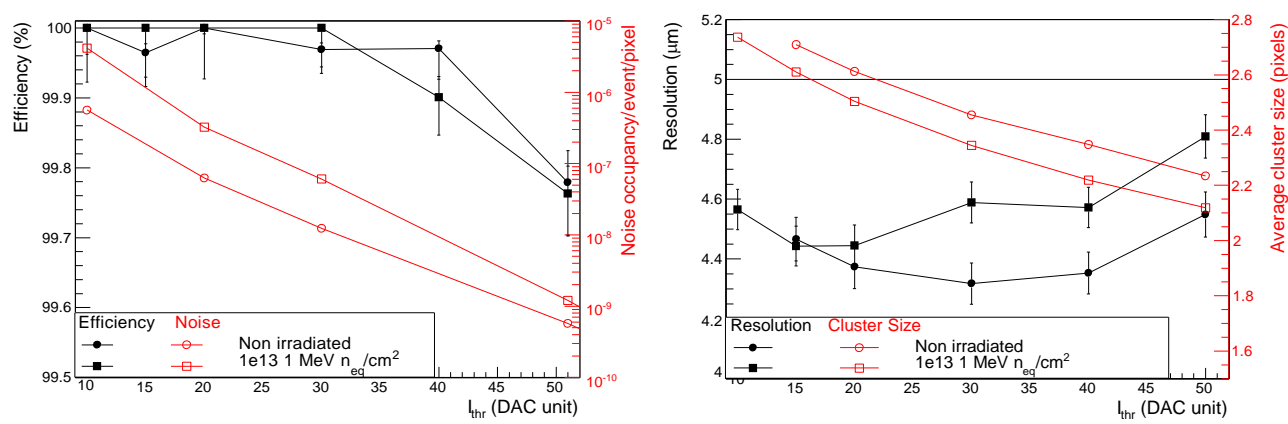

Figure 3: Performance of pALPIDE-1 (sector 2 with $-3 \mathrm{~V}$ reverse substrate bias) to $6 \mathrm{GeV} / c \pi^{-}$. The left panel shows the detection efficiency and fake hit rate of pALPIDE-1 as a function of threshold current. The right panel shows the spatial resolution and average cluster size as a function of threshold current.

irradiated $\left(10^{13} 1 \mathrm{MeV} \mathrm{n}\right.$ eq $\left./ \mathrm{cm}^{2}\right)$ pALPIDE-1 as a function of threshold current. The performance is within the specifications (detection efficiency $>99 \%$ and a fake hit rate $<10^{-5}$ per pixel and event) for a wide range of the threshold current even after irradiation. The right panel shows the spatial resolution and average cluster size as a function of threshold current. The spatial resolution is within the specifications for a wide range of the threshold current.

\section{Detector and physics performance studies}

Performance studies with the upgraded ITS were carried out considering the upgrade scenario presented in the ALICE Upgrade LOI [2]. The left panel of Fig. 4 shows, for example, the expected improvement of the impact parameter resolution in $r-\phi$ with the upgraded ITS. At $500 \mathrm{MeV} / c$, the improvement is a about a factor 3 over the current ITS. Physics performance studies were carried out for heavy flavour, low-mass dielectrons and hypernuclei using specific benchmark channels [4]. The right panel of Fig. 4, for example, shows the expected performance for the measurement of the nuclear modification factor $R_{\mathrm{AA}}$ for $\Lambda_{\mathrm{c}}$ baryons (in the decay channel $\Lambda_{\mathrm{c}} \rightarrow \mathrm{pK}^{-} \pi^{+}$) as a function of $p_{\mathrm{T}}$, for central $\mathrm{Pb}-\mathrm{Pb}$ collisions $(0-20 \%)$ at $\sqrt{s_{N N}}=5.5 \mathrm{TeV}$ with an integrated luminosity $\mathrm{L}_{\mathrm{int}}=10 \mathrm{nb}^{-1}$. This measurement is not accessible with the current ALICE setup. The detailed physics reach for various observables with the upgraded ITS can be found in [4]. 

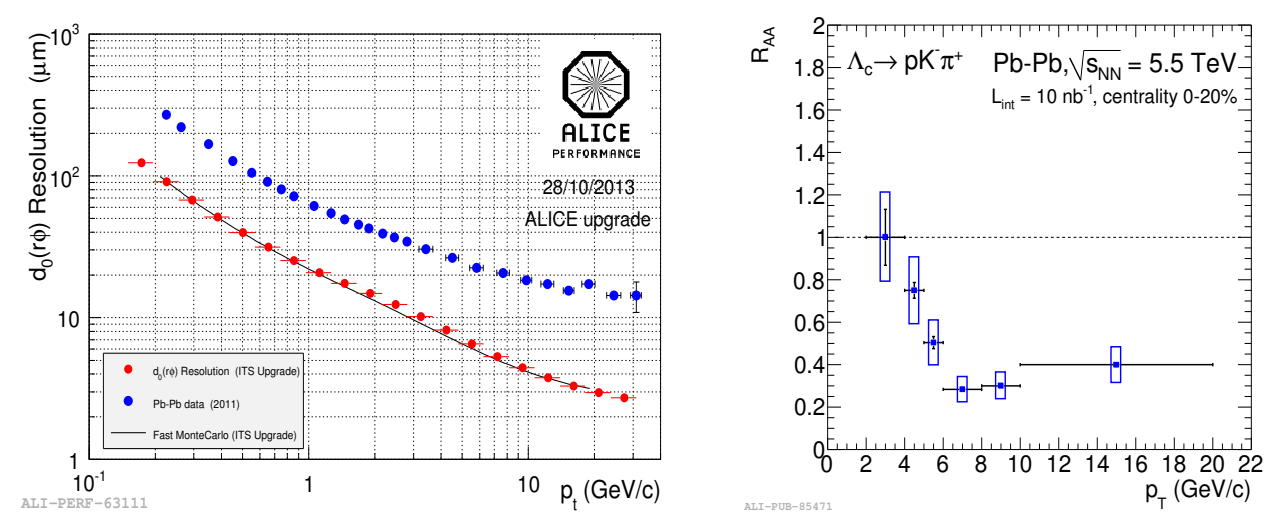

Figure 4: The left panel shows the improvement on the impact parameter resolution with the upgraded ITS over the present ITS. The right panel shows the $\Lambda_{\mathrm{c}} R_{\mathrm{AA}}$ as a function of $p_{\mathrm{T}}$ in central $\mathrm{Pb}-\mathrm{Pb}$ collisions $(0-20 \%)$ for $\mathrm{L}_{\mathrm{int}}=10 \mathrm{nb}^{-1}$.

\section{Conclusions and outlook}

During the last few years, ALICE has carried out a dedicated R \& D programme on the ITS upgrade based on MAPS. The detector layout and the technological aspects were defined and detailed Monte Carlo simulations verified the detector and physics performances. The first full scale prototypes were developed and their performances were studied in detail which satisfied the requirements. Presently, studies are ongoing with the next versions of the full scale prototypes, which will help to converge towards the final design by the beginning of 2016. The production and construction will start in 2016. The detector will be commissioned at surface in 2018/19 and will be installed in the ALICE cavern in 2020. In 2021, the upgraded ALICE detector will start its operations for the high luminosity $\mathrm{Pb}-\mathrm{Pb}$ run.

\section{References}

[1] ALICE Collaboration, The ALICE experiment at the CERN LHC, JINST 3, S08002 (2008).

[2] ALICE Collaboration, Letter of Intent for the Upgrade of the ALICE Experiment, CERN-LHCC-2012-012, LHCC-I-022, 2012.

[3] ALICE Collaboration, Addendum of the Letter Of Intent for the Upgrade of the ALICE Experiment: The Muon Forward Tracker, CERN-LHCC-2013-014, LHCC-I-022-ADD-1, 2013.

[4] ALICE Collaboration, Technical Design Report for the Upgrade of the ALICE Inner Tracking System, J. Phys. G41 (2014) 087002.

[5] L. Greiner et al., A MAPS based vertex detector for the STAR experiment at RHIC, NIM A650, 68-72 (2011).

[6] TowerJazz, TowerJazz 0.18 $\mu \mathrm{m}$ CMOS imaging sensor technology, http://www.jazzsemi.com/.

[7] A. Collu et al., A Monolithic Active Pixel Sensor for the Upgrade of the ALICE ITS, PoS(TIPP2014)337.

[8] P. Yang et al., Low-power priority Address-Encoder and Reset-Decoder data-driven readout for Monolithic Active Pixel Sensors for tracker system, NIM A785 (2015) 61-69. 